\title{
Classification of Tumor Histology via Morphometric Context *
}

\author{
Hang Chang ${ }^{1 \star} \quad$ Alexander Borowsky $^{2} \quad$ Paul Spellman $^{3} \quad$ Bahram Parvin $^{1 \star}$ \\ ${ }^{1}$ Life Sciences Division, Lawrence Berkeley National Laboratory, Berkeley, California, U.S.A \\ * Corresponding authors \{hchang, b_parvin\}@lbl.gov \\ ${ }^{2}$ Center for Comparative Medicine, UC Davis, Davis, California, USA \\ adborowsky@ucdavis.edu \\ ${ }^{3}$ Center for Spatial Systems Biomedicine, Oregon Health Sciences University, Portland, Oregon, U.S.A \\ spel lmap@ohsu.edu
}

\begin{abstract}
Image-based classification of tissue histology, in terms of different components (e.g., normal signature, categories of aberrant signatures), provides a series of indices for tumor composition. Subsequently, aggregation of these indices in each whole slide image (WSI) from a large cohort can provide predictive models of clinical outcome. However, the performance of the existing techniques is hindered as a result of large technical and biological variations that are always present in a large cohort. In this paper, we propose two algorithms for classification of tissue histology based on robust representations of morphometric context, which are built upon nuclear level morphometric features at various locations and scales within the spatial pyramid matching (SPM) framework. These methods have been evaluated on two distinct datasets of different tumor types collected from The Cancer Genome Atlas (TCGA), and the experimental results indicate that our methods are (i) extensible to different tumor types; (ii) robust in the presence of wide technical and biological variations; (iii) invariant to different nuclear segmentation strategies; and (iv) scalable with varying training sample size. In addition, our experiments suggest that enforcing sparsity, during the construction of morphometric context, further improves the performance of the system.
\end{abstract}

\section{Introduction}

Histology sections provide a wealth of information about the tissue architecture that contains multiple cell types at different states of cell cycles. These sections are often

\footnotetext{
* This work was supported by NIH U24 CA1437991 carried out at Lawrence Berkeley National Laboratory under Contract No. DE-AC02$05 \mathrm{CH} 11231$.
}

stained with hematoxylin and eosin (H\&E) stains, which label DNA (e.g., nuclei) and protein contents, respectively, in various shades of color. Abberations in the histology architecture often lead to disease progression. It is desirable to quantify indices associated with these abberations since they can be tested against the clinical outcome, e.g., survival, response to therapy. Even though there are inter- and intra- observer variations [7], a trained pathologist always uses rich content (e.g., various cell types, cellular organization, cell state and health), in context, to characterize tumor architecture.

In this paper, we propose two tissue classification methods based on representations of morphometric context, which are constructed from nuclear morphometric statistics of various locations and scales based on spatial pyramid matching (SPM) [18] and linear spatial pyramid matching (Linear SPM) [28]. Due to the effectiveness of our representations, our methods achieve excellent performance even with small number of training samples across different segmentation strategies and independent datasets of tumors. The performance is further complemented by the fact that one of the methods has a superior result with linear classifiers. These characteristics dramatically improve the (i) effectiveness of our techniques when applied to a large cohort, and (ii) extensibility to other cell-based assays.

Organization of this paper is as follows: Section 2 reviews related works. Sections 3 and 4 describes the details of our proposed mehods and experimental validation. Lastly, section 5 concludes the paper.

\section{Related Work}

For the analysis of the H\&E stained sections, several excellent reviews can be found in $[13,8]$. Fundamentally, the trend has been based either on nuclear segmentation and corresponding morphometric representaion, or patch-based 


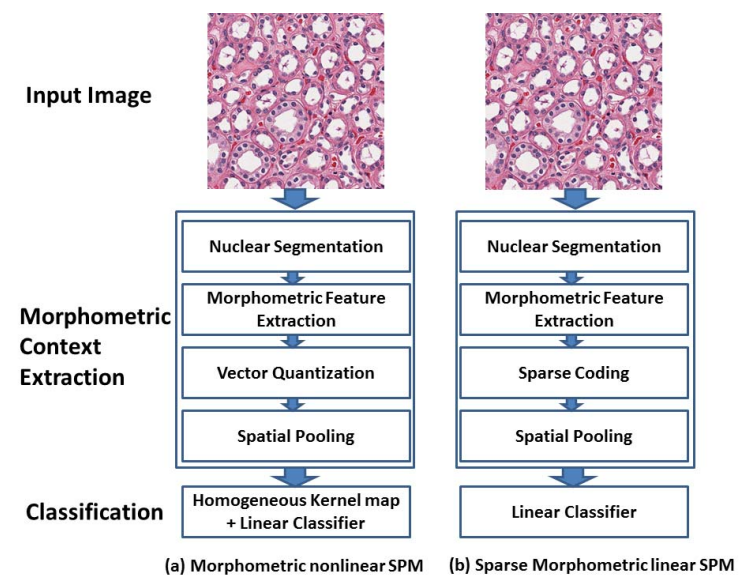

Figure 1. Computational workflows: (a) Morphometric nonlinear kernel SPM; (b) Sparse Morphometric linear SPM. In both approaches, the nuclear segmentation could be based on any of the existing methods.

representation of the histology sections that aids in clinical association. For example, a recent study indicates that detailed segmentation and multivariate representation of nuclear features from H\&E stained sections can predict DCIS recurrence [1] in patients with more than one nuclear grade.

The major challenge for tissue classification is the large amounts of technical and biological variations in the data, which typically results in techniques that are tumor type specific. To overcome this problem, recent studies have focused on either fine tuning human engineered features $[2$, 16, 17], or applying automatic feature learning [19, 14], for robust representation.

In the context of image categorization research, the traditional bag of features $(\mathrm{BoF})$ model has been widely studied and improved through different variations, e.g., modeling of co-occurrence of descriptors based on generative methods [4, 3, 20, 24], improving dictionary construction through discriminative learning $[9,22]$, modeling the spatial layout of local descriptors based on spatial pyramid matching kernel (SPM) [18]. It is clear that SPM has become the major component of the state-of-art systems [10] for its effectiveness in practice.

Pathologists often use "context" to assess the disease state. At the same time, SPM partially captures context because of its hierarchical nature. Motivated by the works of [18, 28], we encode morphometric signatures, at different locations and scales, within the SPM framework. The end results are highly robust and effective systems across multiple tumor types with limited number of training samples.

\section{Approaches}

The computational workflows for the proposed methods are shown in Figure 1, where the nuclear segmentation can be based on any of the existing methods for delineating nu- clei from background. For some tissue images and their corresponding nuclear segmentation, let:

1. $M$ be the total number of segmented nuclei;

2. $N$ be the number of morphometric descriptors extracted from each segmented nucleus, e.g. nuclear size, and nuclear intensity;

3. $\mathbf{X}$ be the set of morphometric descriptors for all segmented nuclei, where $\mathbf{X}=\left[\mathbf{x}_{1}, \ldots, \mathbf{x}_{M}\right]^{\top} \in \mathbb{R}^{M \times N}$.

Our proposed methods are described in detail as follows.

\subsection{Morphometric nonlinear kernel SPM (MK- SPM)}

In this approach, we utilize the nuclear morphometric information within the SPM framework to construct the morphometric context at various locations and scales for tissue image representation and classification. It consists of the following steps:

1. Construct dictionary (D), where $\mathbf{D}=\left[\mathbf{d}_{1}, \ldots, \mathbf{d}_{K}\right]^{\top}$ are the $K$ nuclear morphometric types to be learned by the following optimization:

$$
\begin{aligned}
& \min _{\mathbf{D}, \mathbf{Z}} \sum_{m=1}^{M}\left\|\mathbf{x}_{m}-\mathbf{z}_{m} \mathbf{D}\right\|^{2} \\
& \text { subject to } \operatorname{card}\left(\mathbf{z}_{m}\right)=1,\left|\mathbf{z}_{m}\right|=1, \mathbf{z}_{m} \succeq 0, \forall m
\end{aligned}
$$

where $\mathbf{Z}=\left[\mathbf{z}_{1}, \ldots, \mathbf{z}_{M}\right]^{\top}$ indicates the assignment of the nuclear morphometric type, $\operatorname{card}\left(\mathbf{z}_{m}\right)$ is a cardinality constraint enforcing only one nonzero element of $\mathbf{z}_{m}, \mathbf{z}_{m} \succeq 0$ is a non-negative constraint on the elements of $\mathbf{z}_{m}$, and $\left|\mathbf{z}_{m}\right|$ is the L1-norm of $\mathbf{z}_{m}$. During training, Equation 1 is optimized with respect to both $\mathbf{Z}$ and $\mathbf{D}$; In the coding phase, for a new set of $\mathbf{X}$, the learned $\mathbf{D}$ is applied, and Equation 1 is optimized with respect to $\mathbf{Z}$ only.

2. Construct spatial histogram as the descriptor for the morphometric context for SPM [18]. This is done by repeatedly subdividing an image and computing the histograms of different nuclear morphometric types over the resulting subregions. As a result, the spatial histogram, $H$, is formed by concatenating the appropriately weighted histograms of all nuclear morphometric types at all resolutions. The SPM kernel is then implemented as a single histogram intersection as follows,

$$
\kappa\left(H_{i}, H_{j}\right)=\sum_{q=1}^{Q} \min \left(H_{i}(q), H_{j}(q)\right)
$$

where $H_{i}$ and $H_{j}$ are the spatial histograms for image $I_{i}$ and $I_{j}$, respectively, and $Q$ is the length of the spatial 
histogram. For more details about SPM, please refer to $[18,12]$.

3. Transfer a $\chi^{2}$ support vector machine (SVM) into a linear SVM based on a homogeneous kernel map [26]. In practice, the intersection kernel and $\chi^{2}$ kernel have been found to be the most suitable for histogram representations [28]. In this step, a homogenous kernel map is applied to approximate the $\chi^{2}$ kernels, which enables the efficiency by adopting learning methods for linear kernels, e.g., linear SVM. For more details about the homogeneous kernel map, please refer to [26].

4. Construct multi-class linear SVM for classification. In our implementation, the classifier is trained using the LIBLINEAR [11] package.

\subsection{Sparse Morphometric Linear SPM (SMLSPM)}

Inspired by the work in [28], which indicates that the sparse coding of the SIFT features may serve as a better local descriptor in general image processing tasks, we propose to utilize the sparse coding of the nuclear morphometric information within a linear SPM framework to construct the morphometric context at various locations and scales for tissue image representation and classification. It consists of the following steps:

1. Construct dictionary (D), where $\mathbf{D}=\left[\mathbf{d}_{1}, \ldots, \mathbf{d}_{K}\right]^{\top}$ are the $K$ nuclear morphmetric types to be learned by the following sparse coding optimization:

$$
\begin{aligned}
& \min _{\mathbf{D}, \mathbf{Z}} \sum_{m=1}^{M}\left\|\mathbf{x}_{m}-\mathbf{z}_{m} \mathbf{D}\right\|^{2}+\lambda\left|\mathbf{z}_{m}\right| \\
& \text { subject to }\left\|\mathbf{d}_{k}\right\| \leq 1, \forall k
\end{aligned}
$$

where $\left\|\mathbf{d}_{k}\right\|$ is a unit $L 2$-norm constraint for avoiding trivial solutions, and $\left|\mathbf{z}_{m}\right|$ is the $L 1$-norm enforcing the sparsity of $\mathbf{z}_{m}$. During training, Equation 3 is optimized with respect to both $\mathbf{Z}$ and $\mathbf{D}$; In the coding phase, the learned $\mathbf{D}$ will be applied to a new set of $\mathbf{X}$, with Equation 3 optimized with respect to $\mathbf{Z}$ only.

2. Construct spatial pyramid representation as the descriptor of morphometric context for the linear SPM [28]. Let $\mathbf{Z}$ be the sparse codes calculated through Equation 3 for a descriptor set $\mathbf{X}$. Based on pre-learned and fixed dictionary $\mathbf{D}$, the image descriptor is computed based on a pre-determined pooling function as follows,

$$
\mathbf{f}=\mathbb{P}(\mathbf{Z})
$$

In our implementation, $\mathbb{P}$ is selected to be the $\max$ pooling function on the absolute sparse codes

$$
f_{j}=\max \left\{\left|z_{1 j}\right|,\left|z_{2 j}\right|, \ldots,\left|z_{M j}\right|\right\}
$$

where $f_{j}$ is the $\mathbf{j}$-th element of $\mathbf{f}, z_{i j}$ is the matrix element at $\mathrm{i}$-th row and $\mathrm{j}$-th column of $\mathbf{Z}$, and $M$ is the number of nuclei in the region. The choice of max pooling procedure is justified by biophysical evidence in the visual cortex [25], algorithms in image categorization [28], and our experimental comparison with other common pooling strategies (see Table 7). Similar to the construction of SPM, the pooled features from various locations and scales are then concatenated to form a spatial pyramid representation of the image, and a linear SPM kernel is applied as follows,

$$
\kappa\left(\mathbf{f}_{i}, \mathbf{f}_{j}\right)=\mathbf{f}_{i}^{\top} \mathbf{f}_{j}=\sum_{l=0}^{2} \sum_{s=1}^{2^{l}} \sum_{t=1}^{2^{l}}\left\langle\mathbf{f}_{i}^{l}(s, t), \mathbf{f}_{j}^{l}(s, t)\right\rangle
$$

where $\mathbf{f}_{i}$ and $\mathbf{f}_{j}$ are spatial pyramid representations for image $I_{i}$ and $I_{j}$, respectively, $\left\langle\mathbf{f}_{i}, \mathbf{f}_{j}\right\rangle=\mathbf{f}_{i}^{\top} \mathbf{f}_{j}$, and $\mathbf{f}_{i}^{l}(s, t)$ and $\mathbf{f}_{j}^{l}(s, t)$ are the max pooling statistics of the sparse codes in the $(s, t)$-th segment of image $I_{i}$ and $I_{j}$ in the scale level $l$, respectively.

3. Construct multi-class linear SVM for classification. In our implementation, the classifier is trained using the LIBLINEAR [11] package.

\section{Experiments And Results}

We have evaluated five classification methods on two distinct datasets, curated from (i) Glioblastoma Multiforme (GBM) and (ii) Kidney Renal Clear Cell Carcinoma (KIRC) from The Cancer Genome Atlas (TCGA), which are publicly available from the NIH (National Institute of Health) repository. The five methods are:

1. SMLSPM: the linear SPM that uses linear kernel on spatial-pyramid pooling of morphometric sparse codes;

2. MKSPM: the nonlinear kernel SPM that uses spatialpyramid histograms of morphometric features and $\chi^{2}$ kernels;

3. ScSPM [28]: the linear SPM that uses linear kernel on spatial-pyramid pooling of SIFT sparse codes;

4. KSPM [18]: the nonlinear kernel SPM that uses spatial-pyramid histograms of SIFT features and $\chi^{2}$ kernels;

5. CTSPM: the nonlinear kernel SPM that uses spatialpyramid histograms of color and texture features and $\chi^{2}$ kernels;

In the implementations of SMLSPM and MKSPM, morphometric features were extracted and normalized independently with zero mean and unit variance based on three different segmentation strategies: 


\begin{tabular}{cccc}
\hline \hline Approach & Precision & Recall & F-Measure \\
\hline MRGC & $\mathbf{0 . 7 5}$ & $\mathbf{0 . 8 5}$ & $\mathbf{0 . 7 9 7}$ \\
\hline SRCD & 0.63 & 0.75 & 0.685 \\
\hline OTGR & NA & NA & NA \\
\hline
\end{tabular}

Table 1. Comparison of average segmentation performance among MRGC [6], SRCD [5], and OTGR. Note: 1) the information above is quoted from [6]; 2) the OTGR performance is not listed due to the unavailability of the data used in [6], however, based on our experience with histological sections, simple thresholding methods typically generate less favorable results than sophisticated ones.

1. MRGC [6]: A multi-reference graph cut approach for nuclear segmentation in histology tissue sections;

2. SRCD [5]: A single-reference color decomposition approach for nuclear segmentation in histology tissue sections;

3. OTGR: A simple Otsu thresholding [23] approach for nuclear segmentation in histology tissue sections. In our implementation, nuclear mask was generated by applying Otsu thresholding on gray-scale image, and refined by geometric reasoning [27]. The same refinement was also used in the MRGC and SRCD approaches.

A comparison of the segmentation performance, for the above methods, is quoted from [6], and listed in Table 1, and the computed morphometric features are listed in Table 2 .

In the implementation of ScSPM and KSPM, the dense SIFT features were extracted on $16 \times 16$ patches sampled from each image on a grid with stepsize 8 pixels. In the implementation of CTSPM, color features were extracted in the RGB color space; texture features were extracted via steerable filters [29] with 4 directions and 5 scales ( $\sigma \in\{1,2,3,4,5\})$ on the grayscale image; and the feature vector was a concatenation of texture and mean color on $20 \times 20$ patches.

For both SMLSPM and ScSPM, we fixed the sparse constraint parameter $\lambda$ to be 0.15 , empirically, to achieve the best performance. For MKSPM, KSPM and CTSPM, we used standard K-means clustering for the construction of dictionaries. Additionally, for all five methods, we fixed the level of pyramid to be 3, and used linear SVM for classification. All experimental processes were repeated 10 times with randomly selected training and testing images. The final results were reported as the mean and standard deviation of the classification rates.

\subsection{GBM Dataset}

The GBM dataset contains 3 classes: Tumor, Necrosis, and Transition to Necrosis, which were curated from whole

\begin{tabular}{cccc}
\hline \hline & MRGC & SRCD & OTGR \\
\hline SMLSPM & $92.91 \pm 0.84$ & $\mathbf{9 3 . 5 6} \pm \mathbf{0 . 9 1}$ & $91.03 \pm 1.15$ \\
MKSPM & $91.95 \pm 0.83$ & $\mathbf{9 3 . 3 3} \pm \mathbf{0 . 9 0}$ & $90.94 \pm 0.87$ \\
\hline
\end{tabular}

Table 4. Performance of SMLSPM and MKSPM on the GBM dataset based on three different segmentation approaches, where the number of training images per category was fixed to be 160 , and the dictionary sizes for SMLSPM and MKSPM were fixed to be 1024 and 512, respectively, to achieve optimal performance.

\begin{tabular}{cccc}
\hline \hline & MRGC & SRCD & OTGR \\
\hline SMLSPM & $\mathbf{9 8 . 5 0} \pm \mathbf{0 . 4 2}$ & $98.30 \pm 0.34$ & $97.66 \pm 0.49$ \\
MKSPM & $97.34 \pm 0.48$ & $\mathbf{9 7 . 6 6} \pm \mathbf{0 . 4 5}$ & $95.90 \pm 0.54$ \\
\hline
\end{tabular}

Table 6. Performance of SMLSPM and MKSPM on the KIRC dataset based on three different segmentation approaches, where the number of training images per category was fixed to be 280 , and the dictionary sizes for both SMLSPM and MKSPM were fixed to be 512, to achieve the optimal performance.

slide images (WSI) scanned with a $20 \mathrm{X}$ objective $(0.502$ micron/pixel). Examples can be found in Figure 2. The number of images per category are 628, 428 and 324, respectively. Most images are $1000 \times 1000$ pixels. In this experiment, we trained on 40, 80 and 160 images per category and tested on the rest, with three different dictionary sizes: 256, 512 and 1024. Detailed comparisons are shown in Table 3. For SMLSPM and MKSPM, we also evaluated the performance based on three different segmentation strategies: MRGC, SRCD and OTGR. Detailed comparisons are shown in Table 4.

\subsection{KIRC Dataset}

The KIRC dataset contains 3 classes: Tumor, Normal, and Stromal, which were curated from whole slide images (WSI) scanned with a $40 \mathrm{X}$ objective $(0.252 \mathrm{micron} / \mathrm{pixel})$. Examples can be found in Figure 3. The number of images per category are 568, 796 and 784, respectively. Most images are $1000 \times 1000$ pixels. In this experiment, we trained on 70, 140 and 280 images per category and tested on the rest, with three different dictionary sizes: 256, 512 and 1024. Detailed comparisons are shown in Table 5. For SMLSPM and MKSPM, we also evaluated the performance based on three different segmentation strategies: MRGC, SRCD and OTGR. Detailed comparisons are shown in Table 6

The experiments, conducted on two distinct datasets, demonstrate the following merits of our approach,

1. Extensibility to different tumor types. Tables 3 and 5 indicate that, with the exception of (KIRC; 140 training; Dictionary size 1024), our methods consistently outperform ScSPM, KSPM and CTSPM with different combinations of experimental factors (e.g., train- 


\begin{tabular}{c|c}
\hline \hline Feature & Description \\
\hline Nuclear Size & \#pixels of a segmented nucleus \\
\hline Nuclear Voronoi Size & \#pixels of the voronoi region, where the segmented nucleus resides \\
\hline Aspect Ratio & Aspect ratio of the segmented nucleus \\
\hline Major Axis & Length of Major axis of the segmented nucleus \\
\hline Minor Axis & Length of Minor axis of the segmented nucleus \\
\hline Rotation & Angle between major axis and x axis of the segmented nucleus \\
\hline Bending Energy & Mean squared curvature values along nuclear contour \\
\hline STD Curvature & Standard deviation of absolute curvature values along nuclear contour \\
\hline Abs Max Curvature & Maximum absolute curvature values along nuclear contour \\
\hline Mean Nuclear Intensity & Mean intensity in nuclear region measured in gray scale \\
\hline STD Nuclear Intensity & Standard deviation of intensity in nuclear region measured in gray scale \\
\hline Mean Background Intensity & Mean intensity of nuclear background measured in gray scale \\
\hline STD Background Intensity & Standard deviation of intensity of nuclear background measured in gray scale \\
\hline Mean Nuclear Gradient & Mean gradient within nuclear region measured in gray scale \\
\hline STD Nuclear Gradient & Standard deviation of gradient within nuclear region measured in gray scale \\
\hline
\end{tabular}

Table 2. Morphometric features used in SMLSPM and MKSPM, where the curvature values were computed with $\sigma=2.0$, and the nuclear background is defined to be outside the nuclear region, but inside the bounding box of nuclear boundary.
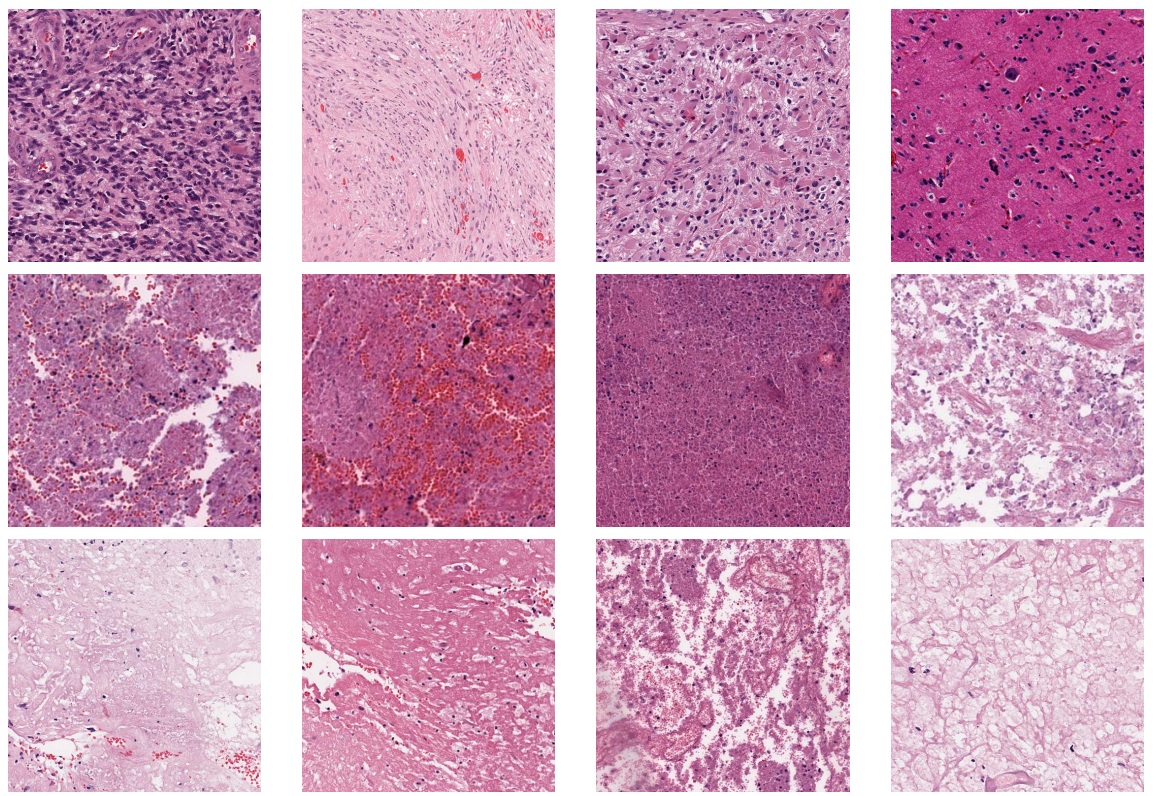

Figure 2. GBM Examples. First row: Tumor; Second row: Transition to necrosis; Third row: Necrosis.

\begin{tabular}{cccc}
\hline \hline & Sqrt & Abs & Max \\
\hline GBM & $92.85 \pm 0.94$ & $90.90 \pm 1.11$ & $\mathbf{9 2 . 9 1} \pm \mathbf{0 . 8 4}$ \\
KIRC & $97.60 \pm 0.49$ & $97.49 \pm 0.38$ & $\mathbf{9 8 . 5 0} \pm \mathbf{0 . 4 2}$ \\
\hline
\end{tabular}

Table 7. Comparison of performance for SMLSPM using different pooling strategies on the GBM and KIRC datasets. For GBM, the number of training images per category was fixed to be 160 , and the dictionary size was fixed to be 1024; for KIRC, the number of training images per category was fixed to be 280 , and the dictionary size was fixed to be 512 . ing sample size, dictionary size). However, KSPM and ScSPM appear to be tumor-type dependent, as KSPM outperforms ScSPM on GBM while ScSPM outperforms KSPM on KIRC. Since GBM and KIRC are two vastly different tumor types with significantly different signatures, we suggest that the consistency in performance assures extensibility to different tumor types.

2. Robustness in the presence of large amounts of technical and biological variations. With respect to the GBM dataset, shown in Table 3, the performance of our methods, based on 40 training samples per cate- 


\begin{tabular}{ccccc}
\hline \hline & Method & DictionarySize=256 & DictionarySize=512 & DictionarySize $=1024$ \\
\hline 160 training & SMLSPM & $92.35 \pm 0.83$ & $92.57 \pm 0.91$ & $\mathbf{9 2 . 9 1} \pm \mathbf{0 . 8 4}$ \\
& MKSPM & $91.85 \pm 0.98$ & $91.95 \pm 0.83$ & $91.76 \pm 0.97$ \\
& ScSPM [28] & $79.58 \pm 0.61$ & $81.29 \pm 0.86$ & $82.36 \pm 1.10$ \\
& KSPM [18] & $85.00 \pm 0.79$ & $86.47 \pm 0.55$ & $86.81 \pm 0.45$ \\
& CTSPM & $78.61 \pm 1.33$ & $78.71 \pm 1.18$ & $78.69 \pm 0.81$ \\
\hline 80 training & SMLSPM & $90.82 \pm 1.28$ & $90.29 \pm 0.68$ & $\mathbf{9 1 . 0 8} \pm \mathbf{0 . 6 9}$ \\
& MKSPM & $89.83 \pm 1.15$ & $89.78 \pm 1.09$ & $89.44 \pm 1.20$ \\
& ScSPM [28] & $77.65 \pm 1.43$ & $78.31 \pm 1.13$ & $81.00 \pm 0.98$ \\
& KSPM [18] & $83.81 \pm 1.22$ & $84.32 \pm 0.67$ & $84.49 \pm 0.34$ \\
& CTSPM & $75.93 \pm 1.18$ & $76.06 \pm 1.52$ & $76.19 \pm 1.33$ \\
\hline 40 training & SMLSPM & $88.05 \pm 1.38$ & $87.88 \pm 1.04$ & $\mathbf{8 8 . 5 4} \pm \mathbf{1 . 4 2}$ \\
& MKSPM & $87.38 \pm 1.38$ & $87.06 \pm 1.52$ & $86.37 \pm 1.73$ \\
& ScSPM [28] & $73.60 \pm 1.68$ & $75.58 \pm 1.29$ & $76.24 \pm 3.05$ \\
& KSPM [18] & $80.54 \pm 1.21$ & $80.56 \pm 1.24$ & $80.46 \pm 0.56$ \\
& CTSPM & $73.10 \pm 1.51$ & $72.90 \pm 1.09$ & $72.65 \pm 1.41$ \\
\hline
\end{tabular}

Table 3. Performance of different methods on the GBM dataset, where SMLSPM and MKSPM were evaluated based on the segmentation method: MRGC [6].
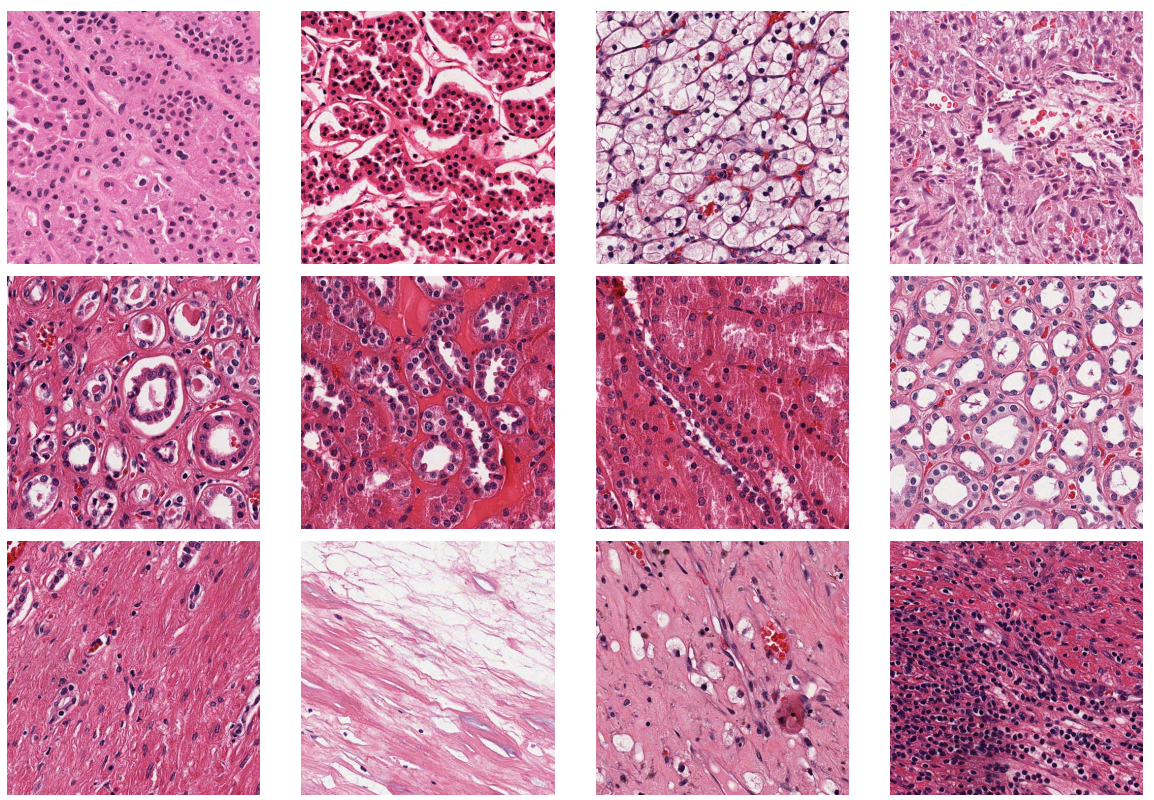

Figure 3. KIRC Examples. First row: Tumor; Second row: Normal; Third row: Stromal.

gory, is better than the performance of ScSPM, KSPM and CTSPM based on 160 training samples per category. With respect to the KIRC dataset, shown in Table 5, the performance of our methods, based on 70 training samples per category, is comparable to the performance of ScSPM, KSPM and CTSPM based on 280 training samples per category. Given the fact that TCGA datasets contain large amount of technical and biological variations $[17,19]$, these results clearly indicate the robustness of our morphometric context representation, which dramatically improved the reliability of our approaches.
3. Invariance to different segmentation algorithms. Tables 4 and 6 indicate that the performance of our approaches are almost invariant to different segmentation algorithms, given the fact that the segmentation performance itself varies a lot, as shown in Table 1. More importantly, even with the simplest segmentation strategy OTGR, SMLSPM outperforms the methods in [28, 18] on both datasets, and MRSPM outperforms the methods in $[28,18]$ on the GBM dataset, while generating comparable results on the KIRC dataset. Given the fact that, in a lot of studies, both nuclear segmentation and tissue classification are necessary components, the 


\begin{tabular}{lcccc}
\hline \hline & Method & DictionarySize $=256$ & DictionarySize $=512$ & DictionarySize $=1024$ \\
\hline 280 training & SMLSPM & $98.15 \pm 0.46$ & $\mathbf{9 8 . 5 0} \pm \mathbf{0 . 4 2}$ & $98.21 \pm 0.44$ \\
& MKSPM & $97.37 \pm 0.49$ & $97.34 \pm 0.48$ & $97.22 \pm 0.50$ \\
& ScSPM [28] & $94.52 \pm 0.44$ & $96.37 \pm 0.45$ & $96.81 \pm 0.50$ \\
& KSPM [18] & $93.55 \pm 0.31$ & $93.76 \pm 0.27$ & $93.90 \pm 0.19$ \\
& CTSPM & $87.45 \pm 0.59$ & $87.95 \pm 0.49$ & $88.53 \pm 0.49$ \\
\hline 140 training & SMLSPM & $97.40 \pm 0.50$ & $\mathbf{9 7 . 9 8} \pm \mathbf{0 . 3 5}$ & $97.35 \pm 0.48$ \\
& MKSPM & $96.56 \pm 0.53$ & $96.54 \pm 0.50$ & $96.41 \pm 0.56$ \\
& ScSPM [28] & $93.46 \pm 0.55$ & $95.68 \pm 0.36$ & $96.76 \pm 0.63$ \\
& KSPM [18] & $92.50 \pm 1.12$ & $93.06 \pm 0.82$ & $93.26 \pm 0.68$ \\
& CTSPM & $86.55 \pm 0.99$ & $86.40 \pm 0.54$ & $86.49 \pm 0.58$ \\
\hline 70 training & SMLSPM & $96.20 \pm 0.85$ & $\mathbf{9 6 . 3 7} \pm \mathbf{0 . 8 5}$ & $96.19 \pm 0.62$ \\
& MKSPM & $95.62 \pm 0.62$ & $95.47 \pm 0.71$ & $95.27 \pm 0.72$ \\
& ScSPM [28] & $91.93 \pm 1.00$ & $93.67 \pm 0.72$ & $94.86 \pm 0.86$ \\
& KSPM [18] & $90.78 \pm 0.98$ & $91.34 \pm 1.13$ & $91.59 \pm 0.97$ \\
& CTSPM & $84.76 \pm 1.32$ & $84.29 \pm 1.53$ & $83.71 \pm 1.42$ \\
\hline
\end{tabular}

Table 5. Performance of different methods on the KIRC dataset, where SMLSPM and MKSPM were evaluated based on the segmentation mehtod: MRGC [6].

use of pre-computed morphometric features dramatically improve the efficiency by avoiding extra feature extraction steps. For example, in our implementation, SIFT costs $1.5 \mathrm{sec} /$ block $($ a block is a $1000 \times 1000$ image decomposed from a whole slide tissue section ). For the whole GBM dataset ( 600,000 blocks), by avoiding SIFT operation, it saves $\sim 10$ days for processing.

4. Scalability of training and high speed testing for SMLSPM. Our experiments show that the morphometric context representation in SMLSPM works well with linear SVMs, which dramatically improves the scalability of training and the speed of testing. This is very important for the analyzing large cohort of whole slide images.

To study the impact of pooling strategies on the SMLSPM method, we also provide an experimental comparison among max pooling and two other common pooling methods, which are defined as follows,

$$
\begin{aligned}
\text { Sqrt }: f_{j} & =\sqrt{\frac{1}{M} \sum_{i=1}^{M} z_{i j}^{2}} \\
\text { Abs }: f_{j} & =\frac{1}{M} \sum_{i=1}^{M}\left|z_{i j}\right|
\end{aligned}
$$

where the meaning of the notations are the same as in Equation 5. As shown in Table 7, the $\max$ pooling strategy outperforms the other two, which is probably due to its robustness to local translations.

The experiments above also indicate a slightly improved performance of SMLSPM over MKSPM; this is probably due to the following factors: i) sparse coding has much less quantization errors than vector quantization; ii) the statistics derived by max pooling are more robust to local translations compared with the average pooling in the histogram representation.

\section{Conclusion and Future Work}

In this paper, we proposed two spatial pyramid matching approaches based on morphometric features and morphometric sparse code, respectively, for tissue image classification. By modeling the context of the morphometric information, these methods outperform traditional ones which were typically based on pixel- or patch-level features. The most encouraging results of this paper are that, our methods are highly i) extensible to different tumor types; ii) robust in the presence of large amounts of technical and biological variations; iii) invariant to different segmentation algorithms; and iv) scalable to extremely large training and testing sample sizes. Due to i) the effectiveness of our morphometric context representations; and ii) the important role of cellular context for the study of different cell assays, proposed methods are suggested to be extendable to image classification tasks for different cell assays. Future work will be focused on accelerating the sparse coding through the sparse auto encoder [15], utilizing supervised dictionary learning [21] for possible improvement, and further validating our methods on other tissue types and other cell assays.

\section{References}

[1] D. Axelrod, N. Miller, H. Lickley, J. Qian, W. ChristensBarry, Y. Yuan, Y. Fu, and J. Chapman. Effect of quantitative 
nuclear features on recurrence of ductal carcinoma in situ (dcis) of breast. In Cancer Informatics, 4:99-109, 2008. 2

[2] R. Bhagavatula, M. Fickus, W. Kelly, C. Guo, J. Ozolek, C. Castro, and J. Kovacevic. Automatic identification and delineation of germ layer components in $h \&$ e stained images of teratomas derived from human and nonhuman primate embryonic stem cells. In ISBI, pages 1041-1044, 2010. 2

[3] O. Boiman, E. Shechtman, and M. Irani. In defense of nearest-neighbor based image classification. In Proceedings of the Conference on Computer Vision and Pattern Recognition, pages 1-8, 2008. 2

[4] A. Bosch, A. Zisserman, and X. Muñoz. Scene classification using a hybrid generative/discriminative approach. IEEE Transactions on Pattern Analysis and Machine Intelligence, 30(4):712-727, Apr. 2008. 2

[5] H. Chang, G. Fontenay, J. Han, G. Cong, F. Baehner, J. Gray, P. Spellman, and B. Parvin. Morphometric analysis of TCGA Gliobastoma Multiforme. BMC Bioinformatics, 12(1), 2011. 4

[6] H. Chang, L. Loss, P. Spellman, A. Borowsky, and B. Parvin. Batch-invariant nuclear segmentation in whole mount histology sections. In ISBI, pages 856-859, 2012. 4, 6, 7

[7] L. Dalton, S. Pinder, C. Elston, I. Ellis, D. Page, W. Dupont, and R. Blamey. Histolgical gradings of breast cancer: linkage of patient outcome with level of pathologist agreements. Modern Pathology, 13(7):730-735, 2000. 1

[8] C. Demir and B. Yener. Automated cancer diagnosis based on histopathological images: A systematic survey, 2009. 1

[9] M. Elad and M. Aharon. Image denoising via sparse and redundant representations over learned dictionaries. IEEE Transactions on Image Processing, 15(12):3736-3745, Dec. 2006. 2

[10] M. Everingham, L. Van Gool, C. K. I. Williams, J. Winn, and A. Zisserman. The PASCAL Visual Object Classes Challenge 2012 (VOC2012) Results. http://www.pascalnetwork.org/challenges/VOC/voc2012/workshop/index.html. 2

[11] R.-E. Fan, K.-W. Chang, C.-J. Hsieh, X.-R. Wang, and C.-J. Lin. LIBLINEAR: A library for large linear classification. Journal of Machine Learning Research, 9:1871-1874, 2008. 3

[12] K. Grauman and T. Darrell. The pyramid match kernel: discriminative classification with sets of image features. In Proceedings of the IEEE International Conference on Computer Vision, volume 2, pages 1458 - 1465, 2005. 3

[13] M. Gurcan, L. Boucheron, A. Can, A. Madabhushi, N. Rajpoot, and Y. Bulent. Histopathological image analysis: a review. IEEE Transactions on Biomedical Engineering, 2:147-171, 2009. 1

[14] C. Huang, A. Veillard, N. Lomeine, D. Racoceanu, and L. Roux. Time efficient sparse analysis of histopathological whole slide images. Computerized medical imaging and graphics, 35(7-8):579-591, 2011. 2

[15] K. Kavukcuoglu, M. Ranzato, and Y. LeCun. Fast inference in sparse coding algorithms with applications to object recognition. Technical Report CBLL-TR-2008-12-01, Computational and Biological Learning Lab, Courant Institute, NYU, 2008. 7

[16] J. Kong, L. Cooper, A. Sharma, T. Kurk, D. Brat, and J. Saltz. Texture based image recognition in microscopy images of diffuse gliomas with multi-class gentle boosting mechanism. In ICASSAP, pages 457-460, 2010. 2

[17] S. Kothari, J. Phan, A. Osunkoya, and M. Wang. Biological interpretation of morphological patterns in histopathological whole slide images. In ACM Conference on Bioinformatics, Computational Biology and Biomedicine, 2012. 2, 6

[18] S. Lazebnik, C. Schmid, and J. Ponce. Beyond bags of features: Spatial pyramid matching for recognizing natural scene categories. In Proceedings of the Conference on Computer Vision and Pattern Recognition, pages 2169-2178, 2006. 1, 2, 3, 6, 7

[19] Q. Le, J. Han, J. Gray, P. Spellman, A. Borowsky, and B. Parvin. Learning invariant features from tumor signature. In $I S B I$, pages 302-305, 2012. 2, 6

[20] F.-F. Li and P. Perona. A bayesian hierarchical model for learning natural scene categories. In Proceedings of the Conference on Computer Vision and Pattern Recognition, pages 524-531, Washington, DC, USA, 2005. IEEE Computer Society. 2

[21] J. Mairal, F. Bach, J. Ponce, G. Sapiro, and A. Zisserman. Supervised dictionary learning. In NIPS, 2008. 7

[22] F. Moosmann, B. Triggs, and F. Jurie. Randomized clustering forests for building fast and discriminative visual vocabularies. In NIPS, 2006. 2

[23] N. Otsu. A threshold selection method from gray-level histograms. IEEE Transactions on Systems, Man and Cybernetics, 9(1):62-66, 1979. 4

[24] P. Quelhas, F. Monay, J.-M. Odobez, D. Gatica-Perez, T. Tuytelaars, and L. Van Gool. Modeling scenes with local descriptors and latent aspects. In Proceedings of the IEEE International Conference on Computer Vision, ICCV '05, pages 883-890, Washington, DC, USA, 2005. IEEE Computer Society. 2

[25] T. Serre, L. Wolf, and T. Poggio. Object recognition with features inspired by visual cortex. In Proceedings of the Conference on Computer Vision and Pattern Recognition, volume 2, pages 994-1000, 2005. 3

[26] A. Vedaldi and A. Zisserman. Efficient additive kernels via explicit feature maps. IEEE Transactions on Pattern Analysis and Machine Intelligence, 34(3):480-492, 2012. 3

[27] Q. Wen, H. Chang, and B. Parvin. A Delaunay triangulation approach for segmenting clumps of nuclei. In $I S B I$, pages 9-12, 2009. 4

[28] J. Yang, K. Yu, Y. Gong, and T. Huang. Linear spatial pyramid matching using sparse coding for image classification. In Proceedings of the Conference on Computer Vision and Pattern Recognition, pages 1794-1801, 2009. 1, 2, 3, 6, 7

[29] R. A. Young and R. M. Lesperance. The gaussian derivative model for spatial-temporal vision. I. Cortical Model. Spatial Vision, 2001:3-4, 2001. 4 